\title{
Apolipoprotein D Upregulation in Alzheimer's Disease but Not Frontotemporal Dementia
}

\author{
Surabhi Bhatia ${ }^{1}$ (D) Woojin Scott Kim ${ }^{1,2,3} \cdot$ Claire E. Shepherd ${ }^{2,3} \cdot$ Glenda M. Halliday $^{1,2,3}$
}

Received: 22 August 2018 / Accepted: 11 November 2018 / Published online: 22 November 2018

(C) The Author(s) 2018

\begin{abstract}
Frontotemporal dementia (FTD) and Alzheimer's disease (AD) are the two common forms of dementia. FTD syndromes are characterized by lobar atrophy (frontotemporal lobar degeneration or FTLD) and the presence of either cellular TDP43 (FTLD-TDP), tau (FTLD-tau), or FUS aggregates, while extracellular $\beta$-amyloid plaques and hyperphosphorylated tau tangles develop in AD. Oxidative stress can induce these pathological modifications in disease models, and is thought to play a role in these syndromes. Apolipoprotein D (apoD) is a glial-expressed lipocalin known to protect against oxidative stress, with increased levels in $\mathrm{AD}$, supporting a protective role. The expression of apoD has not been studied in FTLD. This study assesses apoD expression in FTLD-TDP and FTLD-tau in comparison to AD and controls. It also analyzes the effect of apoD on TARDBP (TDP43 gene) and $\beta$-amyloid precursor protein (APP). The expression of apoD was analyzed by Western blotting in FTLD-TDP, FTLD-tau, AD, and control post-mortem brain tissue. An apoD-overexpressing cell model was used to study the impact of increased apoD on APP and TARDBP expression. We confirm that apoD expression was increased in $\mathrm{AD}$ but surprisingly it was not affected in either of the two main pathological forms of FTLD. Under oxidative stress conditions, apoD had no effect on TDP43 expression but it did decrease APP expression. This suggests that apoD does not act as a neuroprotective factor in FTLD in the same way as in $\mathrm{AD}$. This could contribute to the more rapid degeneration observed in FTLD.
\end{abstract}

Keywords Apolipoprotein D · Oxidative stress $\cdot$ Neurodegeneration $\cdot$ Frontotemporal dementia $\cdot$ TDP43

$\begin{array}{ll}\text { Abbreviations } \\ \text { apoD } & \text { Apolipoprotein D } \\ \text { FTLD } & \text { Frontotemporal lobar degeneration } \\ \text { AD } & \text { Alzheimer's disease } \\ \text { PD } & \text { Parkinson's disease } \\ \text { PSP } & \text { Progressive supranuclear palsy } \\ \text { CBD } & \text { Corticobasal degeneration }\end{array}$

Electronic supplementary material The online version of this article (https://doi.org/10.1007/s12031-018-1217-9) contains supplementary material, which is available to authorized users.

Surabhi Bhatia

surabhi.bhatia@sydney.edu.au

1 Central Clinical School and Brain and Mind Centre, Faculty of Medicine and Health Sciences, The University of Sydney, 94 Mallet Street, Camperdown, NSW, Australia

2 Neuroscience Research Australia, Sydney, NSW, Australia

3 School of Medical Sciences, University of New South Wales, Sydney, NSW, Australia
GGT Globular glial taupathies

TBS Tris-buffered saline

FBS Fetal bovine serum

\section{Introduction}

Apolipoprotein D (apoD) is a $29 \mathrm{kDa}$ highly conserved lipocalin known for its antioxidant and neuroprotective functions (He et al. 2009; Tsukamoto et al. 2013; Dassati et al. 2014). ApoD is known to be upregulated in astrocytes during aging (Loerch et al. 2008; de Magalhaes et al. 2009) and in various neurological disorders including schizophrenia, bipolar disorder (Thomas et al. 2001; Mahadik et al. 2002), stroke (Rassart et al. 2000), Alzheimer's disease (AD) (Terrisse et al. 1998; Belloir et al. 2001; Glockner and Ohm 2003; Bhatia et al. 2013), Parkinson's disease (PD) (Ordonez et al. 2006), and Niemann-Pick's C disease (Suresh et al. 1998). ApoD knockout mouse models have provided evidence that the loss of apoD is associated with motor and cognitive deficits 
(Ganfornina et al. 2008; Bajo-Grañeras et al. 2011a). The loss of apoD is known to disrupt proteostasis machinery and induce oxidative and inflammatory damage (Thomas et al. 2003; Sanchez et al. 2015). It has been demonstrated that apoD helps to maintain neuronal homeostasis by combatting lipid peroxidation (Ganfornina et al. 2008).

Frontotemporal lobar degeneration (FTLD) and AD are two major forms of dementia. Neuropathologically, TDP43 and tau protein aggregates are present in the majority of FTLD cases (IR and Manuela 2016) while extracellular $\beta$ amyloid plaques produced from amyloid precursor protein (APP) and intracellular hyperphosphorylated tau tangles are the hallmarks of $\mathrm{AD}$ (Bloom 2014). Oxidative stress is one of the major factors associated with both of these pathological forms of dementia. Oxidative stress response genes are dysregulated in both TDP43-overexpressing mice (Tsuiji et al. 2017) and human brain post-mortem tissues with FTLD pathology (Schweitzer et al. 2006). Moreover, under induced oxidative stress, cellular TDP43 undergoes phosphorylation and $\mathrm{C}$ terminal fragmentation, characteristic of pathological TDP43 in FTLD (Iguchi et al. 2012). Similarly, increased levels of lipid and protein oxidation products are evident in AD (Lyras et al. 1997; Montine et al. 2005) and these are known to modulate $\beta$-amyloid production (Tong et al. 2005; Tamagno et al. 2008; Tan et al. 2013a).

ApoD is upregulated in brain astrocytes and CSF of AD patients (Terrisse et al. 1998; Belloir et al. 2001; Bhatia et al. 2013) and is known to colocalize with $\beta$-amyloid plaques (Desai et al. 2005). Studies have demonstrated that the apoD homolog in Drosophila (GLaz) protects against $\beta$-amyloidinduced cytotoxicity (Muffat et al. 2008). In addition, transgenic $\mathrm{AD}$ mouse models with loss of apoD function exhibit twice the amount of hippocampal $\beta$-amyloid plaque load along with alterations in $\beta$-amyloid-generating proteins ( $\mathrm{Li}$ et al. 2015). Increasing evidence suggests the antioxidant and anti-inflammatory role of apoD (Ganfornina et al. 2008; Bajo-Grañeras et al. 2011b; Dassati et al. 2014). We therefore hypothesized that apoD plays a neuroprotective role in FTLD; and to test our hypothesis, we analyzed the expression of apoD in FTLD and compared that to AD and controls. As oxidative stress is known to induce pathological modifications in TDP43 and APP, we also assessed the effect of apoD on $A P P$ and TARDBP (TAR DNA-binding protein) gene expression, responsible for pathological aggregates in AD and FTLD respectively.

Our data suggests that unlike $\mathrm{AD}$, apoD is not increased in FTLD. The cell models suggest that under oxidative stress, apoD protects against increased expression of $A P P$ while it has no effect on TARDBP, which is responsible for TDP43 expression. Therefore, this suggests that apoD expression is differentially regulated in FTLD and AD and that it is possible that apoD is unable to protect against oxidative stress in FTLD.

\section{Material and Methods}

\section{Human Brain Tissue}

Frozen post-mortem brain tissue was obtained with approval from Sydney Brain Bank and NSW Brain Tissue Resource Centre as part of the NSW Brain Banks. Brain tissue from the inferior temporal and superior frontal cortex from 18 FTLD cases, 7 AD cases (5 for inferior temporal region), and 11 controls, all without other neuropathological abnormalities, were used in this study. In particular, both the FTLD and control cases were selected for having no or low AD neuropathologic change. Ten FTLD cases had TDP-43 aggregates (FTLD-TDP) with Type A, B, and C pathologies (Tan et al. 2013b), and 8 FTLD cases had tau aggregates (FTLD-tau) with 4R subtype pathologies (Kovacs 2018). Demographic information for each group is provided in Table 1. Ethics approval for the study was from the University of New South Wales Human Research Ethics Committee.

\section{Sample Preparation}

Tris-buffered saline (TBS), SDS-soluble and SDS-insoluble proteins were serially extracted from $100 \mathrm{mg}$ of fresh-frozen tissue from each brain region, as previously described (Bhatia et al. 2013; Murphy et al. 2013). Briefly, tissue was homogenized in ten volumes of TBS homogenization buffer $(20 \mathrm{mM}$ Tris, $150 \mathrm{mM} \mathrm{NaCl}, \mathrm{pH} 7.4,5 \mathrm{mM}$ EDTA, $0.02 \%$ sodium azide) containing protease inhibitor cocktail (Roche) using Qiagen tissue lyser $(3 \times 30 \mathrm{sec}, 30 \mathrm{~Hz}$ cycles $)$, followed by centrifugation at $100,000 \times g$ for $1 \mathrm{~h}$ at $4{ }^{\circ} \mathrm{C}$, with supernatant collected as the TBS-soluble fraction containing cytosolic proteins. The pellet was resuspended in SDS solubilization buffer (TBS homogenization buffer containing 5\% SDS) using $3 \times$ $30 \mathrm{sec}, 30 \mathrm{~Hz}$ cycles with Qiagen Tissue Lyser, and centrifuged at $100,000 \times g$ for $30 \mathrm{~min}$ at $25^{\circ} \mathrm{C}$, with supernatant collected as the SDS-soluble fraction containing membraneassociated proteins. The pellet obtained by SDS fraction was homogenized in $5 \mathrm{M}$ guanidine hydrochloride $(\mathrm{gHCl})$ and mixed in a mixer at room temperature for $4 \mathrm{~h}$. The lysate was further centrifuged at $100,000 \times g$ for $30 \mathrm{~min}$ at $25^{\circ} \mathrm{C}$, with supernatant collected as the SDS-insoluble ( $\mathrm{gHCl}$ soluble) fraction. Protein concentration was measured using a bicinchoninic acid assay (Pierce BCA Protein Assay Kit, Thermo Scientific), according to the manufacturer's instructions. Samples were stored at $-80^{\circ} \mathrm{C}$ until use.

\section{Immunoblotting}

Equal concentrations of TBS fraction of the protein extracts $(15 \mu \mathrm{g})$ were heated with sample buffer $(3.2 \%$ SDS, $32 \%$ glycerol, $0.16 \%$ bromophenol blue, $100 \mathrm{mM}$ Tris-HCl, 
Table 1 Demographic and pathological details of control, FTLD-TDP43, FTLD-tau, and $\mathrm{AD}$ cohorts. Values are given as mean \pm standard deviation for age and post-mortem delay (PMI)

\begin{tabular}{lllcl}
\hline Group & $N$ & Age & PMI (h) & Gender (M/F) \\
\hline Control & 11 & $79.5 \pm 12.1$ & $16.3 \pm 6.5$ & $5 / 6$ \\
FTLD-TDP43 & 10 & $72.9 \pm 13$ & $24 \pm 8.7$ & $5 / 5$ \\
FTLD-tau & 8 & $73.9 \pm 5.8$ & $12.5 \pm 9$ & $4 / 4$ \\
AD & 7 (superior frontal) & $73.7 \pm 7.5$ & $2.3 \pm 0.7$ & $3 / 4$ \\
\hline
\end{tabular}

pH 6.8, 8\% 2-mercaptoethanol $w / v$ ) and separated on Bio-Rad Criterion Stain-free 4-20\% SDS-PAGE gels. The gels were activated for $1 \mathrm{~min}$ using Bio-Rad chemiDoc MP imaging system prior to transfer of proteins to a $0.45-\mu \mathrm{m}$ PVDF membrane. The membranes were imaged for total protein using Bio-Rad chemiDoc MP imaging system. Subsequently, the membranes were blocked with $5 \%$ milk powder in TBST for $1 \mathrm{~h}$ at room temperature and incubated overnight in apoD primary antibody (Santacruz, sc-373965, 1:2000) prior to protein detection using horseradish peroxidase-conjugated secondary antibodies (Bio-Rad) with enhanced chemiluminescence (Amersham ECL Plus Western Blot Detection System, GE Healthcare). The protein band in each gel lane was normalized to total protein using Bio-Rad image lab software.

\section{Cell Culture}

U87-MG cell line (astroglioma) obtained from ATCC was used as apoD is highly expressed in astrocytic cells in vivo. To obtain an apoD-overexpressing stable cell line, the cells were transfected with human apoD plasmid (obtained from OriGENE) using Lipofectamine 3000 as per manufacture's protocol and then maintained in EMEM media with $10 \%$ fetal bovine serum (FBS) and $0.5 \mathrm{mg} / \mathrm{ml}$ Geneticin for 2 weeks. After the initial selection process, the cells were maintained in media with $0.25 \mathrm{mg} / \mathrm{ml}$ Geneticin. The cells were plated in a six well dish at the density of $1 \times 10^{5}$ cells $/ \mathrm{ml}$ and treated with $300 \mu \mathrm{M}$ of $\mathrm{H}_{2} \mathrm{O}_{2}$ for $24 \mathrm{~h}$ to induce oxidative stress. The experiments were repeated in triplicates for three times.

\section{RNA Extraction and qPCR}

RNA was extracted from the cells using Relia Prep RNA cell Miniprep system (promega) as per manufacture's protocol. Analysis of $A P P$ and TARDBP expression was performed using iScript cDNA synthesis Kit and SSO advanced SYBR green mix on Bio-Rad CFX connect system. Amplification was carried out with 40 cycles of $94{ }^{\circ} \mathrm{C}$ for $15 \mathrm{~s}$ and $60{ }^{\circ} \mathrm{C}$ for $1 \mathrm{~min}$. The details of the primers used for the study is as follows-APP (F: 5'CCGCTGCTTAGTTGGTGAGTTTGT-3' and R: 5'-ACGGTGTGCCAGTGAAGATGAGTT-3'), TARDBP (F 5'-CGGCCTAGCGGGAAAAGTAA-3' and R:5'TGGAAACTGGGCTGTAACCG-3'), $\beta$-actin (F: 5'GAATTCTGGCCACGGCTGCTTCCAGCT-3', and R: 5'-
AAGCTTTTTCGTGGATGCCACAGGACT-3') $5.8 S$ forward 5'-GGTGGATCACTCGGCTCGT-3', and R: 5'GCAAGTGCGTTCGAAGTGTC-3', Cyclophilin A (F: 5'AGGGTTCCTGCTTTCACAGA-3'and R: 5'-GTCT TGGCAGTGCAGATGAA-3').

All gene expression was normalized to the housekeeper genes $\beta$-actin, Cyclophilin A, and 5.8S. A no-template control was included for each PCR amplification. The level of expression for each gene was calculated using the comparative threshold cycle $(\mathrm{CT})$ value method with the formula $2^{-\Delta \Delta \mathrm{Ct}}$ (where $\Delta \Delta \mathrm{CT}=\Delta \mathrm{CT}$ sample $-\Delta \mathrm{CTreference)}$.

\section{Data Analysis}

All statistical analyses for post-mortem apoD expression in human brain were performed using SPSS statistical software using univariate analysis covarying for age, sex, and gender, with a $p$ value $<0.05$ considered significant. The relative expression of apoD in human brain tissue is expressed as a percentage of control. The analysis for cell models was completed using Welch's $t$ test on Prism Graphpad, $p$ value $<0.05$ was considered significant.

\section{Results}

\section{ApoD Expression in FTLD and AD}

The relative expression of apoD in post-mortem brain tissue of FTLD and AD samples was analyzed in the highly affected superior frontal cortex (SFC) and inferior temporal cortex (ITC) using western blotting. There was no significant difference in soluble apoD levels in FTLD compared to controls in either of the regions analyzed [C vs FTLD-TDP43-SFC; $p=$ 0.262 , ITC; $p=0.490]$. This is in contrast to AD where we confirm previous findings of significantly increased apoD protein levels [C vs AD-SFC; $p=0.005$, ITC; $p=0.002$ ] (Belloir et al. 2001; Glockner and Ohm 2003; Desai et al. 2005; Bhatia et al. 2013) (Fig. 1a, b). Comparison of FTLD cases with either TDP43 or tau pathology found no significant differences in the relative soluble apoD levels even in the most affected superior frontal cortex between these groups [FTLDTDP43 vs FTLD-tau, $p=0.367$ ] (Fig. 1a). These data suggest that there are significant differences in the expression of TBSsoluble apoD between FTLD and AD, two different 


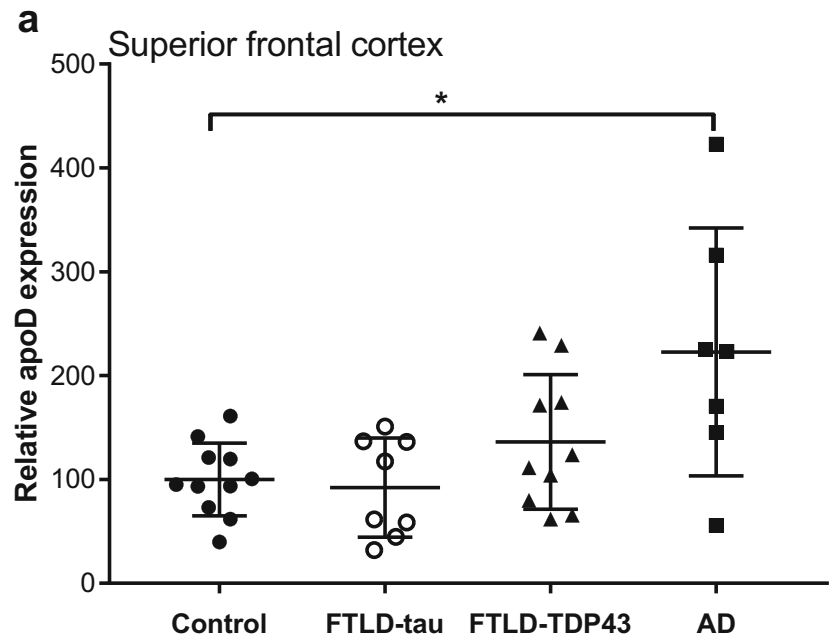

b

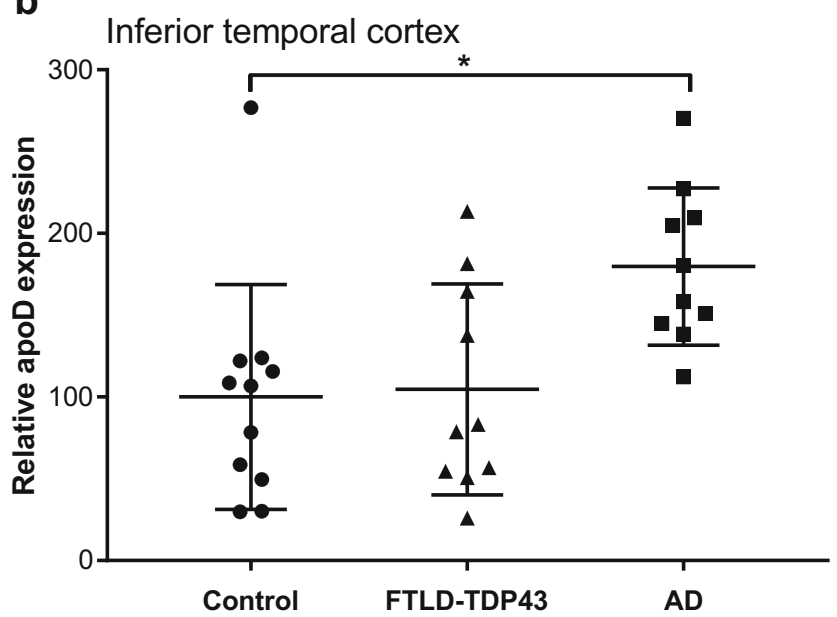

Fig. 1 Relative expression of ApoD in TBS-soluble fraction in a the superior frontal cortex and $\mathbf{b}$ inferior temporal cortex. Data represents mean \pm SD. Significance is at $* p<0.05$

neurodegenerative dementias. As the expression of apoD has not been previously studied in FTLD, we also analyzed the SDS-soluble and SDS-insoluble ( $\mathrm{gHCl}$ soluble) fraction from the superior frontal cortex in the diseased cohorts. There was no significant difference in apoD expression in either SDSsoluble (Suppl. Fig. 1a,b,c) or gHCL-soluble fractions (Suppl. Fig. 2) in any of the disease groups when compared to controls. Furthermore, we did not detect any specific higher molecular weight aggregates in $\mathrm{gHCl}$ fractions in either FTLD or AD compared to controls (Suppl. Fig. 2).

\section{Effect of apoD on TARDBP and APP Expression Under Oxidative Stress}

As mentioned previously, TARDBP is the gene encoding the protein that deposits in the pathological aggregates in FTLDTDP. Therefore, we analyzed the effect of apoD on TARDBP. We found that apoD overexpression has no impact on
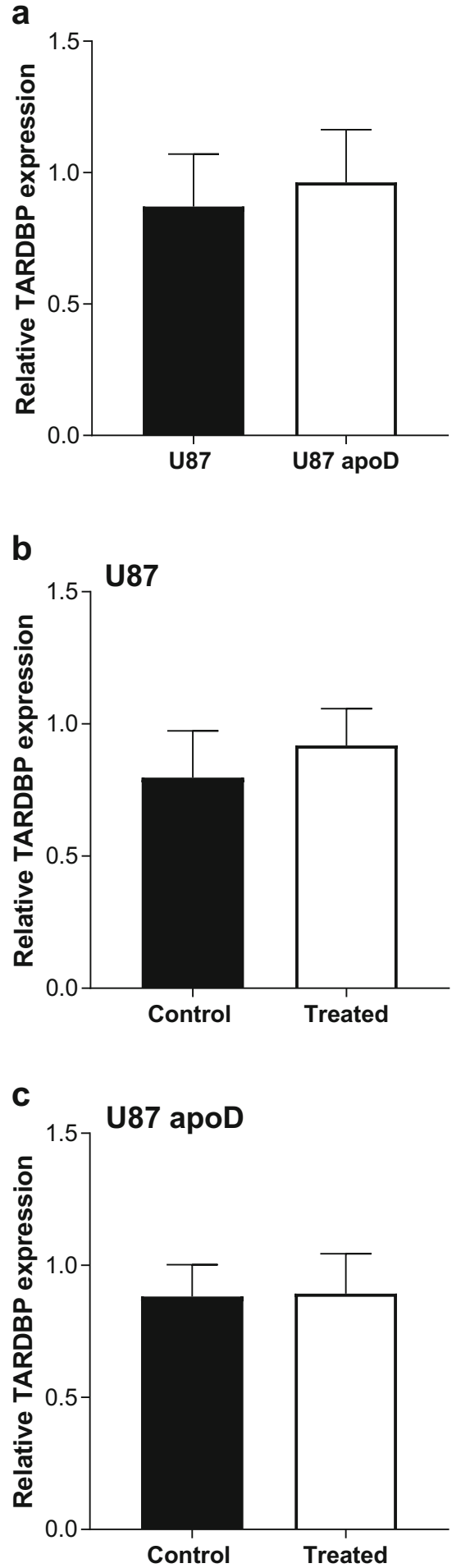

Fig. 2 Relative expression of TARDBP in a U87- and apoDoverexpressing cell line, $\mathbf{b}$ in U87 cells, and $\mathbf{c}$ U87 apoD cells under control vs treated ( $300 \mathrm{~mm} \mathrm{H} 2 \mathrm{O} 2$ for $24 \mathrm{~h}$ ) conditions. Data represents mean \pm SD. of $n=3$ for 3 repeats

endogenous TARDBP expression (Fig. 2a). To determine if increased apoD could impact AD pathology, we analyzed the expression of amyloid precursor protein $(A P P)$ in apoDoverexpressing U87 cells. We observed no change in 
expression of $A P P$ in apoD-overexpressing U87 cells (Fig. 3a) suggesting that the expression of apoD is unlikely to impact on astrocytic $\beta$-amyloid production.

Oxidative stress induces pathological modifications in TDP43 (Cohen et al. 2012; Iguchi et al. 2012) and results in increased $\beta$-amyloid production (Paola et al. 2000).
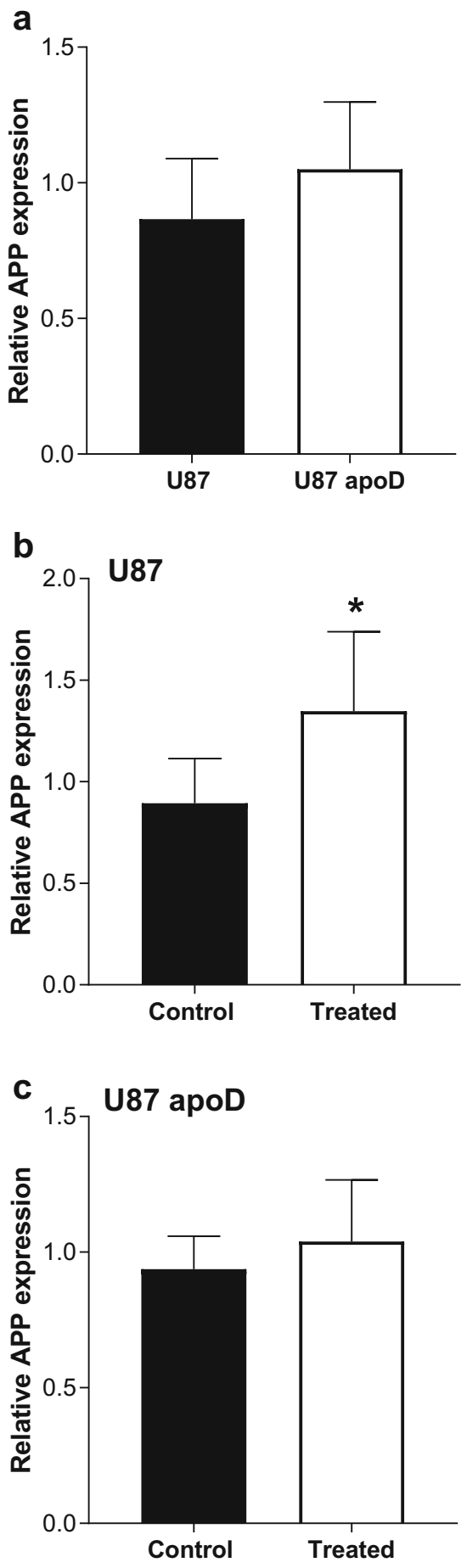

Fig. 3 Relative expression of APP in (a) U87- and apoD-overexpressing cell line, b in U87 cells, and c U87 apoD cells under control vs treated ( $300 \mathrm{~mm} \mathrm{H} 2 \mathrm{O} 2$ for $24 \mathrm{~h}$ ) conditions. Data represents mean $\pm \mathrm{SD}$ of $n=3$ for 3 repeats. Significance is at $* p<0.05$
Therefore, we analyzed the effect of apoD on TARDBP and APP expression under oxidative stress. Different concentration and exposure times of hydrogen peroxide $\left(\mathrm{H}_{2} \mathrm{O}_{2}\right)$ were used to induce oxidative stress to the cells, and based on cell viability assays we used the $300 \mu \mathrm{M} \mathrm{H}_{2} \mathrm{O}_{2}, 24-\mathrm{h}$ treatment to induce oxidative stress (suppl. Fig. 3). We found that apoD has no effect on $T A R D B P$ expression under oxidative stress (Fig. 2b, c). However, we found that in the presence of $\mathrm{H}_{2} \mathrm{O}_{2}$, $A P P$ expression was significantly upregulated in U87 cells but this effect was not evident in apoD-overexpressing U87 cells (Fig. 3b, c).

\section{Discussion}

This study analyzes the expression of apoD in FTLD, AD, and control brain tissue. As apoD expression in FTLD has not been studied previously, we analyzed the apoD expression in TBS soluble (cytosolic), SDS soluble, and SDS insoluble ( $\mathrm{gHCl}$ soluble) in FTLD. We found that unlike AD, there was no change in cytosolic apoD expression in either FTLDTDP or FTLD-tau compared to age-matched controls. Furthermore, there was no difference in the expression of cytosolic apoD between FTLD-TDP and FTLD-tau, two major pathological forms of FTLD (Fig. 1a, b). We also found no difference in apoD expression in SDS-soluble and $\mathrm{gHCl}$ soluble fraction in either FTLD-TDP or FTLD-tau. Furthermore, in contrast to our previous study which has reported apoD dimers in gHCL-soluble fraction (with pathological aggregates) in the hippocampal region in $\mathrm{AD}$ (Bhatia et al. 2013), we found no specific apoD aggregates in either FTLD groups or $\mathrm{AD}$ in the superior frontal cortex. This suggests that apoD may be present as a dimer only in the highly affected hippocampal region in $\mathrm{AD}$ and not in the superior frontal cortex. These results indicate that in contrast to AD where cytosolic apoD expression is increased along with presence of apoD dimers in the highly affected hippocampal region, apoD expression is unaltered and apoD does not seem to aggregate in the most affected superior frontal region in FTLD.

A major difference between AD and FTLD is the deposition of extracellular $\beta$-amyloid pathology found in AD, while both have abnormal intracellular protein deposits in the remaining cortical neurons (Ferrer et al. 2014; Irwin et al. 2015; Tan et al. 2015). Therefore, $\beta$-amyloid-induced oxidative stress or the presence of $\beta$-amyloid itself may be responsible for increased apoD in $\mathrm{AD}$ as suggested previously (Martinez et al. 2012).

Apart from extracellular $\beta$-amyloid aggregates, $\mathrm{AD}$ is also characterized by the presence of intracellular tau deposition. Intracellular tau pathology also underlies a proportion of cases with FTLD (Arai et al. 2006; Leyton and Hodges 2010; Goedert et al. 2012). Our data demonstrate that tau deposition in FTLD does not increase apoD protein expression, 
suggesting that neuronal tau accumulation of itself does not cause astrocytic upregulation of apoD. Also, the type of tau accumulations in AD versus FTLD differs, with both 3 repeat and 4 repeat tau isoforms incorporated into the neuritic pathology in $\mathrm{AD}$, while only 4 repeat tau isoforms are incorporated into the neuritic pathology of the FTLD-tau cases analyzed in this study (Goedert et al. 1989; Yoshida 2006; Dickson et al. 2011). Astrocytes have 4 repeat tau isoforms (Nishimura et al. 1997; Arai et al. 2001) and it may be that increasing 3 repeat tau protein is a trigger in AD compared with the FTLD.

Alternatively, it may be different in the potential reactivity of astrocytes in these two neurodegenerative dementias that affect apoD expression. Astrocytic apoptosis correlates with the degree of atrophy in FTLD (Broe et al. 2004) and the early loss of astrocytes in FTLD may explain the lack of any potential increase in apoD protein levels in these cases. In fact, reduced astrocytic apoD results in stress-induced astrocytic apoptotic cell death (Bajo-Grañeras et al. 2011a) which may further contribute to the degeneration of these cells in FTLD.

Furthermore, our data suggests that oxidative stress increases $\beta$-amyloid production in astrocytes, and that such increased $\beta$-amyloid production can be ameliorated by increasing the expression of apoD, in agreement with previous studies (Desai et al. 2005; Martinez et al. 2012; Li et al. 2015). Overall, this data is consistent with an increase expression of astrocytic apoD protein in association with increased oxidation and $\beta$-amyloid production in AD. We also found that in the presence of $\mathrm{H}_{2} \mathrm{O}_{2}$, there was no difference in TDP43 expression in U87 cells (Fig. 2), a finding in line with the concept that oxidative stress impacts on TDP43 in a neuron-specific way (Cacabelos et al. 2016), and also in line with our data showing no change in apoD in FTLD-TDP.

Previous studies using apoD knock out mice have shown that apoD specifically protects against oxidation of lipids in the brain while it has no effect on protein oxidation (Ganfornina et al. 2008). A large number of studies have shown increased lipid peroxidation in the AD brain (Pratico et al. 1998; Williams et al. 2006; Fukuda et al. 2009). In comparison, only one study has assessed lipid peroxidation in FTLD and reports that F2 isoprostane (lipid peroxidation marker) levels are not increased in FTLD compared to controls (Yao et al. 2003). The absence of excessive oxidation of brain lipids in FTLD suggests that clearance of lipid peroxidation products may be well preserved in FTLD and therefore, apoD levels remain unaltered. Overall, the data show that the neuroprotective apoD is increased in $\mathrm{AD}$ brain tissue but not in FTLD, suggesting significantly different reactions to oxidative stress in these two forms of dementia- the apoD response in AD appears to be somewhat neuroprotective, while its unchanging level in FTLD may exacerbate the neurodegeneration.
Acknowledgements We thank Heidi Cartwright for her assistance with the preparation of figures.

Funding Information This work was supported by funding from the National Health and Medical Research Council-Australian Research Council (NHMRC-ARC) Dementia Research Development Fellowship, GNT1108882 (to SB). Tissues were received from the New South Wales Tissue Resource Centre at the University of Sydney and the Sydney Brain Bank at Neuroscience Research Australia which are supported by The University of New South Wales, Neuroscience Research Australia and Schizophrenia Research Institute.

\section{Compliance with Ethical Standards}

Conflict of Interest The authors declare that they have no conflict of interest.

Ethics Approval This research project was approved by the Human Research Ethics Committees of the University of New South Wales. Tissues were selected from a neuropathological series collected by the New South Wales Brain Bank through regional brain donor programs in Sydney, Australia. The brain donor programs hold approval from the Human Research Ethics Committees of the University of New South Wales and comply with the statement on human experimentation issued by the National Health and Medical Research Council of Australia.

Open Access This article is distributed under the terms of the Creative Commons Attribution 4.0 International License (http:// creativecommons.org/licenses/by/4.0/), which permits unrestricted use, distribution, and reproduction in any medium, provided you give appropriate credit to the original author(s) and the source, provide a link to the Creative Commons license, and indicate if changes were made.

\section{References}

Arai T, Ikeda K, Akiyama H, Shikamoto Y, Tsuchiya K, Yagishita S, Beach T, Rogers J, Schwab C, McGeer P (2001) Distinct isoforms of tau aggregated in neurons and glial cells in brains of patients with Pick's disease, corticobasal degeneration and progressive supranuclear palsy. Acta Neuropathol 101:167-173

Arai T, Hasegawa M, Akiyama H, Ikeda K, Nonaka T, Mori H, Mann D, Tsuchiya K, Yoshida M, Hashizume Y, Oda T (2006) TDP-43 is a component of ubiquitin-positive tau-negative inclusions in frontotemporal lobar degeneration and amyotrophic lateral sclerosis. Biochem Biophys Res Commun 351:602-611. https://doi.org/10. 1016/j.bbrc.2006.10.093

Bajo-Grañeras R, Ganfornina MD, Martín-Tejedor E, Sanchez D (2011a) Apolipoprotein D mediates autocrine protection of astrocytes and controls their reactivity level, contributing to the functional maintenance of paraquat-challenged dopaminergic systems. Glia 59:15511566. https://doi.org/10.1002/glia.21200

Bajo-Grañeras R, Sanchez D, Gutierrez G, González C, Do Carmo S, Rassart E, Ganfornina MD (2011b) Apolipoprotein D alters the early transcriptional response to oxidative stress in the adult cerebellum. J Neurochem 117:949-960. https://doi.org/10.1111/j.14714159.2011.07266.x

Belloir B, Kovari E, Surini-Demiri M, Savioz A (2001) Altered apolipoprotein D expression in the brain of patients with Alzheimer disease. J Neurosci Res 64:61-69. https://doi.org/ 10.1002/jnr. 1054

Bhatia S, Jenner AM, Li H, Ruberu K, Spiro AS, Shepherd CE, Kril JJ, Kain N, Don A, Garner B (2013) Increased apolipoprotein D dimer 
formation in Alzheimer's disease hippocampus is associated with lipid conjugated diene levels. J Alzheimers Dis 35:475-486. https://doi.org/10.3233/jad-122278

Bloom GS (2014) Amyloid-beta and tau: the trigger and bullet in Alzheimer disease pathogenesis. JAMA Neurol 71:505-508. https://doi.org/10.1001/jamaneurol.2013.5847

Broe M, Kril J, Halliday GM (2004) Astrocytic degeneration relates to the severity of disease in frontotemporal dementia. Brain 127:2214 2220. https://doi.org/10.1093/brain/awh250

Cacabelos D, Ayala V, Granado-Serrano AB, Jové M, Torres P, Boada J, Cabré R, Ramírez-Núñez O, Gonzalo H, Soler-Cantero A, Serrano JCE, Bellmunt MJ, Romero MP, Motilva MJ, Nonaka T, Hasegawa M, Ferrer I, Pamplona R, Portero-Otín M (2016) Interplay between TDP-43 and docosahexaenoic acid-related processes in amyotrophic lateral sclerosis. Neurobiol Dis 88:148-160. https://doi.org/10.1016/ j.nbd.2016.01.007

Cohen TJ, Hwang AW, Unger T, Trojanowski JQ, Lee VM (2012) Redox signalling directly regulates TDP-43 via cysteine oxidation and disulphide cross-linking. EMBO J 31:1241-1252. https://doi.org/10. 1038/emboj.2011.471

Dassati S, Waldner A, Schweigreiter R (2014) Apolipoprotein D takes center stage in the stress response of the aging and degenerative brain. Neurobiol Aging 35:1632-1642. https://doi.org/10.1016/j. neurobiolaging.2014.01.148

de Magalhaes JP, Curado J, Church GM (2009) Meta-analysis of agerelated gene expression profiles identifies common signatures of aging. Bioinformatics 25:875-881. https://doi.org/10.1093/ bioinformatics/btp073

Desai PP, Ikonomovic MD, Abrahamson EE, Hamilton RL, Isanski BA, Hope CE, Klunk WE, DeKosky ST, Kamboh MI (2005) Apolipoprotein D is a component of compact but not diffuse amyloid-beta plaques in Alzheimer's disease temporal cortex. Neurobiol Dis 20:574-582. https://doi.org/10.1016/j.nbd.2005.04. 012

Dickson DW, Kouri N, Murray ME, Josephs KA (2011) Neuropathology of frontotemporal lobar degeneration-tau (FTLD-tau). J Mol Neurosci 45:384-389. https://doi.org/10.1007/s12031-011-9589-0

Ferrer I, López-González I, Carmona M, Arregui L, Dalfó E, TorrejónEscribano B, Diehl R, Kovacs GG (2014) Glial and neuronal tau pathology in tauopathies: characterization of disease-specific phenotypes and tau pathology progression. J Neuropathol Exp Neurol 73: 81-97. https://doi.org/10.1097/nen.0000000000000030

Fukuda M et al (2009) Elevated levels of 4-hydroxynonenal-histidine Michael adduct in the hippocampi of patients with Alzheimer's disease. Biomed Res 30:227-233

Ganfornina MD, Do Carmo S, Lora JM, Torres-Schumann S, Vogel M, Allhorn M, Gonzlez C, Bastiani MJ, Rassart E, Sanchez D (2008) Apolipoprotein $\mathrm{D}$ is involved in the mechanisms regulating protection from oxidative stress. Aging Cell 7:506-515. https://doi.org/10. $1111 /$ j.1474-9726.2008.00395.x

Glockner F, Ohm TG (2003) Hippocampal apolipoprotein D level depends on Braak stage and APOE genotype. Neuroscience 122: $103-110$

Goedert M, Spillantini MG, Potier MC, Ulrich J, Crowther RA (1989) Cloning and sequencing of the cDNA encoding an isoform of microtubule-associated protein tau containing four tandem repeats: differential expression of tau protein mRNAs in human brain. EMBO J 8:393-399

Goedert M, Ghetti B, Spillantini MG (2012) Frontotemporal dementia: implications for understanding Alzheimer disease. Cold Spring Harb Perspect Med 2:a006254. https://doi.org/10.1101/ cshperspect.a006254

He X, Jittiwat J, Kim JH, Jenner AM, Farooqui AA, Patel SC, Ong WY (2009) Apolipoprotein D modulates F2-isoprostane and 7ketocholesterol formation and has a neuroprotective effect on organotypic hippocampal cultures after kainate-induced excitotoxic injury. Neurosci Lett 455:183-186. https://doi.org/10.1016/j.neulet. 2009.03.038

Iguchi Y, Katsuno M, Takagi S, Ishigaki S, Niwa JI, Hasegawa M, Tanaka F, Sobue G (2012) Oxidative stress induced by glutathione depletion reproduces pathological modifications of TDP-43 linked to TDP-43 proteinopathies. Neurobiol Dis 45:862-870. https://doi.org/10. 1016/j.nbd.2011.12.002

IR M, Manuela N (2016) Molecular neuropathology of frontotemporal dementia: insights into disease mechanisms from postmortem studies. J Neurochem 138:54-70. https://doi.org/10.1111/jnc.13588

Irwin DJ, Cairns NJ, Grossman M, McMillan CT, Lee EB, van Deerlin VM, Lee VMY, Trojanowski JQ (2015) Frontotemporal lobar degeneration: defining phenotypic diversity through personalized medicine. Acta Neuropathol 129:469-491. https://doi.org/10.1007/ s00401-014-1380-1

Kovacs GG (2018) Chapter 25 - Tauopathies. In: Kovacs GG, Alafuzoff I (eds) Handbook of clinical neurology, vol 145. Elsevier, Amsterdam, pp 355-368. https://doi.org/10.1016/B978-0-12802395-2.00025-0

Leyton C, Hodges J (2010) Frontotemporal dementias: recent advances and current controversies. Ann Indian Acad Neurol 13:74-80. https://doi.org/10.4103/0972-2327.74249

Li H, Ruberu K, Muñoz SS, Jenner AM, Spiro A, Zhao H, Rassart E, Sanchez D, Ganfornina MD, Karl T, Garner B (2015) Apolipoprotein D modulates amyloid pathology in APP/PS1 Alzheimer's disease mice. Neurobiol Aging 36:1820-1833. https:// doi.org/10.1016/j.neurobiolaging.2015.02.010

Loerch PM, Lu T, Dakin KA, Vann JM, Isaacs A, Geula C, Wang J, Pan Y, Gabuzda DH, Li C, Prolla TA, Yankner BA (2008) Evolution of the aging brain transcriptome and synaptic regulation. PLoS One 3: e3329. https://doi.org/10.1371/journal.pone.0003329

Lyras L, Cairns NJ, Jenner A, Jenner P, Halliwell B (1997) An assessment of oxidative damage to proteins, lipids, and DNA in brain from patients with Alzheimer's disease. J Neurochem 68:2061-2069

Mahadik SP, Khan MM, Evans DR, Parikh VV (2002) Elevated plasma level of apolipoprotein D in schizophrenia and its treatment and outcome. Schizophr Res 58:55-62

Martinez E, Navarro A, Ordonez C, Del Valle E, Tolivia J (2012) Amyloid-beta25-35 induces apolipoprotein D synthesis and growth arrest in HT22 hippocampal cells. J Alzheimers Dis 30:233-244. https://doi.org/10.3233/jad-2012-112102

Montine TJ, Montine KS, McMahan W, Markesbery WR, Quinn JF, Morrow JD (2005) F2-isoprostanes in Alzheimer and other neurodegenerative diseases. Antioxid Redox Signal 7:269-275. https:// doi.org/10.1089/ars.2005.7.269

Muffat J, Walker DW, Benzer S (2008) Human ApoD, an apolipoprotein up-regulated in neurodegenerative diseases, extends lifespan and increases stress resistance in Drosophila. Proc Natl Acad Sci U S A 105:7088-7093. https://doi.org/10.1073/pnas.0800896105

Murphy KE, Cottle L, Gysbers AM, Cooper AA, Halliday GM (2013) ATP13A2 (PARK9) protein levels are reduced in brain tissue of cases with Lewy bodies. Acta Neuropathol Commun 1:11. https:// doi.org/10.1186/2051-5960-1-11

Nishimura T, Ikeda K, Akiyama H, Arai T, Kondo H, Okochi M, Furiya Y, Mori H, Oda T, Kato M, Iseki E (1997) Glial tau-positive structures lack the sequence encoded by exon 3 of the tau protein gene. Neurosci Lett 224:169-172. https://doi.org/10.1016/s03043940(97)00161-4

Ordonez C, Navarro A, Perez C, Astudillo A, Martinez E, Tolivia J (2006) Apolipoprotein D expression in substantia nigra of Parkinson disease. Histol Histopathol 21:361-366. https://doi.org/ $10.14670 / \mathrm{hh}-21.361$

Paola D, Domenicotti C, Nitti M, Vitali A, Borghi R, Cottalasso D, Zaccheo D, Odetti P, Strocchi P, Marinari UM, Tabaton M, Pronzato MA (2000) Oxidative stress induces increase in intracellular amyloid beta-protein production and selective activation of betal 
and betaII PKCs in NT2 cells. Biochem Biophys Res Commun 268: 642-646. https://doi.org/10.1006/bbrc.2000.2164

Pratico D, V MYL, Trojanowski JQ, Rokach J, Fitzgerald GA (1998) Increased F2-isoprostanes in Alzheimer's disease: evidence for enhanced lipid peroxidation in vivo. FASEB J 12:1777-1783

Rassart E, Bedirian A, Do Carmo S, Guinard O, Sirois J, Terrisse L, Milne R (2000) Apolipoprotein D. Biochim Biophys Acta 1482:185-198. https://doi.org/10.1016/S0167-4838(00)00162-X

Sanchez D, Bajo-Grañeras R, Del Caño-Espinel M, Garcia-Centeno R, Garcia-Mateo N, Pascua-Maestro R, Ganfornina MD (2015) Aging without apolipoprotein D: molecular and cellular modifications in the hippocampus and cortex. Exp Gerontol 67:19-47. https://doi. org/10.1016/j.exger.2015.04.003

Schweitzer K, Decker E, Zhu L, Miller RE, Mirra SS, Spina S, Ghetti B, Wang M, Murrell J (2006) Aberrantly regulated proteins in frontotemporal dementia. Biochem Biophys Res Commun 348: 465-472. https://doi.org/10.1016/j.bbrc.2006.07.113

Suresh S, Yan Z, Patel RC, Patel YC, Patel SC (1998) Cellular cholesterol storage in the Niemann-Pick disease type $\mathrm{C}$ mouse is associated with increased expression and defective processing of apolipoprotein D. J Neurochem 70:242-251

Tamagno E, Guglielmotto M, Aragno M, Borghi R, Autelli R, Giliberto L, Muraca G, Danni O, Zhu X, Smith MA, Perry G, Jo DG, Mattson MP, Tabaton M (2008) Oxidative stress activates a positive feedback between the gamma- and beta-secretase cleavages of the betaamyloid precursor protein. J Neurochem 104:683-695. https://doi. org/10.1111/j.1471-4159.2007.05072.x

Tan JL, Li QX, Ciccotosto GD, Crouch PJ, Culvenor JG, White AR, Evin $\mathrm{G}$ (2013a) Mild oxidative stress induces redistribution of BACE1 in non-apoptotic conditions and promotes the amyloidogenic processing of Alzheimer's disease amyloid precursor protein. PLoS One 8: e61246. https://doi.org/10.1371/journal.pone.0061246

Tan RH, Shepherd CE, Kril JJ, McCann H, McGeachie A, McGinley C, Affleck A, Halliday GM (2013b) Classification of FTLD-TDP cases into pathological subtypes using antibodies against phosphorylated and non-phosphorylated TDP43. Acta Neuropathol Commun 1:33. https://doi.org/10.1186/2051-5960-1-33

Tan RH, Kril JJ, Fatima M, McGeachie A, McCann H, Shepherd C, Forrest SL, Affleck A, Kwok JBJ, Hodges JR, Kiernan MC, Halliday GM (2015) TDP-43 proteinopathies: pathological identification of brain regions differentiating clinical phenotypes. Brain 138:3110-3122. https://doi.org/10.1093/ brain/awv220

Terrisse L et al (1998) Increased levels of apolipoprotein D in cerebrospinal fluid and hippocampus of Alzheimer's patients. J Neurochem 71:1643-1650

Thomas EA, Dean B, Pavey G, Sutcliffe JG (2001) Increased CNS levels of apolipoprotein D in schizophrenic and bipolar subjects: implications for the pathophysiology of psychiatric disorders. Proc Natl Acad Sci U S A 98:4066-4071. https://doi.org/10.1073/pnas. 071056198

Thomas EA, George RC, Sutcliffe JG (2003) Apolipoprotein D modulates arachidonic acid signaling in cultured cells: implications for psychiatric disorders. Prostaglandins Leukot Essent Fat Acids 69: 421-427

Tong Y, Zhou W, Fung V, Christensen MA, Qing H, Sun X, Song W (2005) Oxidative stress potentiates BACE1 gene expression and Abeta generation. J Neural Transm (Vienna 1996) 112:455-469. https://doi.org/10.1007/s00702-004-0255-3

Tsuiji H, Inoue I, Takeuchi M, Furuya A, Yamakage Y, Watanabe S, Koike M, Hattori M, Yamanaka K (2017) TDP-43 accelerates agedependent degeneration of interneurons. Sci Rep 7:14972. https:// doi.org/10.1038/s41598-017-14966-w

Tsukamoto K, Mani DR, Shi J, Zhang S, Haagensen DE, Otsuka F, Guan J, Smith JD, Weng W, Liao R, Kolodgie FD, Virmani R, Krieger M (2013) Identification of apolipoprotein D as a cardioprotective gene using a mouse model of lethal atherosclerotic coronary artery disease. Proc Natl Acad Sci U S A 110:17023-17028. https://doi.org/ 10.1073/pnas. 1315986110

Williams TI, Lynn BC, Markesbery WR, Lovell MA (2006) Increased levels of 4-hydroxynonenal and acrolein, neurotoxic markers of lipid peroxidation, in the brain in mild cognitive impairment and early Alzheimer's disease. Neurobiol Aging 27:1094-1099. https://doi. org/10.1016/j.neurobiolaging.2005.06.004

Yao Y, Zhukareva V, Sung S, Clark CM, Rokach J, Lee VMY, Trojanowski JQ, Pratico D (2003) Enhanced brain levels of 8,12iso-iPF2alpha-VI differentiate AD from frontotemporal dementia. Neurology 61:475-478

Yoshida M (2006) Cellular tau pathology and immunohistochemical study of tau isoforms in sporadic tauopathies. Neuropathology 26: 457-470. https://doi.org/10.1111/j.1440-1789.2006.00743.x 